\title{
Comparison of endoscopic based diagnosis with Helicobacter urease test for Helicobacter pylori infection
}

\author{
N. A. Adu-Aryee ${ }^{1}$, L. Aabakken², F. Dedey ${ }^{1}$, J. Nsaful ${ }^{1}$ and W. Kudzi ${ }^{3 *}$
}

\begin{abstract}
Background: Helicobacter pylori is an important risk factor for gastritis, peptic ulcers and gastric cancer. The prevalence in developed countries is lower than $40 \%$ but higher than $80 \%$ in some developing countries. It is $75 \%$ in Ghana. The Helicobacter urease test (HUT) is performed at endoscopy and gives an accurate diagnosis. The HUT is not routinely done at our facility and presumption of $H$. pylori is made based on endoscopic findings and $H$. pylori eradication prescribed, as the incidence in the general population is presumed high. Is this endoscopic diagnosis sufficient for diagnosing and treating $H$. pylori? We aimed to assess the feasibility of an endoscopic based H. pylori diagnosis and its accuracy using a HUT as the gold standard in consecutive patients.
\end{abstract}

Methods: Seventy-six consecutive adult patients with dyspepsia were assessed by upper gastrointestinal endoscopy. A clinical diagnosis of H. pylori or not was made. Biopsy samples were collected for HUT. H. pylori was diagnosed if HUT was positive. The results were then compared.

Results: Median age of patients was 45.0 years. H. pylori prevalence detected by HUT was $51.3 \%$ (95 \% Cl 40.0-63.0). Sensitivity of endoscopic diagnosis of H. pylori was $71.8 \%$ (95 \% Cl 55.1-85.0) and specificity was $37.8 \%(95 \% \mathrm{Cl}$ 22.5-55.2). There was no association between clinical findings (73.7 \%) and HUT (26.3\%) (OR $=0.80$; $[95 \% \mathrm{Cl} 0.24-$ $2.64], p=0.682)$. There was also no association between endoscopic diagnosis ( $71.8 \%)$ and HUT (28.2\%), $(\mathrm{OR}=1.55$; $95 \%$ Cl 0.59-4.06, $p=0.373)$.

Conclusion: Helicobacter pylori infection was not as high as that published in earlier reports. The endoscopic diagnosis alone is not sufficient to make a diagnosis of H. pylori.

Keywords: Helicobacter pylori, Helicobacter urease test, Endoscopic diagnosis

\section{Background}

Helicobacter pylori $(H$. pylori) infection is the most common cause of bacterial infection worldwide $[1,2]$ infecting more than $50 \%$ of the world's population predominantly in the developing countries [3]. H. pylori is an important risk factor for gastritis, peptic ulcers, and gastric cancer likely due to the extensive inflammation in the stomach [3, 4]. H. pylori has been associated with the development of two forms of cancer and has led to the

\footnotetext{
*Correspondence: wkudzi@yahoo.com

${ }^{3}$ Centre for Tropical Clinical Pharmacology and Therapeutics, School of Medicine and Dentistry, College of Health Sciences, P.O. Box GP 4236, Accra, Ghana

Full list of author information is available at the end of the article
}

World Health Organization (WHO) classifying it as the only bacterial Class I carcinogen. Unless treated, colonization usually persists for life, indicating that $H$. pylori is well adapted to the gastric environment.

Prevalence of $H$. pylori infection varies among countries and within different racial groups within the same country. The highest rates of infection are generally associated with low socio-economic status, crowding, poor sanitation and unclean water supplies [5].

In the developed countries the prevalence of $H$. pylori is lower than $40 \%$ but in some developing countries more than $50 \%$ of children are infected by the age of 10 years with prevalence of infection rising to more than $80 \%$ in young adults [6]. In Ivory Coast, $55 \%$ of children 
aged $<10$ years have been reported to be infected, while in northern Nigeria and Gambia, 50 \% of children under 5 years are infected [7]. A recent study in Soweto found $46 \%$ of children at 1 year and $100 \%$ of children at 12 years to be infected with $H$. pylori [7]. The prevalence of $H$. pylori in dyspeptic patients has been reported to be $75 \%$ in Ghana [3, 4], $91.3 \%$ in Ivory Coast [8] and $72-91 \%$ in Nigeria $[9,10]$.

The diagnosis of $H$. pylori infection relies on the various testing methods which may be invasive or non-invasive. There are invasive techniques such as histological examination, bacterial cultures, rapid urease test, use of deoxyribonucleic acid probes, and polymerase chain reaction (PCR) analysis all of which require an endoscopy and a biopsy. Non-invasive techniques such as urea breath tests, immunoglobulin G and M serology, Stool antigen test, gastric juice PCR, and urinary excretion of N15 ammonia do not require endoscopy. These various methods have been found to be sensitive and specific [1] but require skilled persons to perform them correctly. H. pylori urease test is fast, simple and does not need highly experienced laboratory staff to accurately identify $H$. pylori infection in the endoscopy unit within few hours of the procedure [11].

Epigastric pain, dyspepsia, haematemesis and melaena have been the commonest reasons for endoscopy. Chronic duodenal ulcer, acute gastritis, duodenitis and oesophagitis were the commonest diagnoses in Korle $\mathrm{Bu}$ Teaching Hospital from January 1995 to December 2002 [3]. H. pylori plays an important role in the aetiopathogenesis of peptic ulcer disease among Ghanaians [3, 4]. Previous studies in Korle Bu Teaching Hospital using urease test confirmed positive results in $75 \%$ of all biopsy specimen [3]. Early diagnosis and treatment can lead to the eradication of $H$. pylori which will reduce $H$. pylori related peptic ulcer disease. $H$. pylori testing schemes in Accra are diverse and non-systematic. Upper gastrointestinal (GI) endoscopy is an established method of investigation in Korle $\mathrm{Bu}$ Teaching Hospital for $\mathrm{H}$. pylori diagnosis using antral biopsies for histology. However, it is not routinely performed. The current practice in Korle $\mathrm{Bu}$ Teaching Hospital involves making a diagnosis based on endoscopic findings. Occasionally biopsies may be taken for histologic conformation of $H$. pylori infection.

This study determined the current incidence of $H$. pylori infection in Korle Bu Teaching Hospital and assessed the feasibility of an endoscopic based H. pylori diagnosis and its accuracy using a Helicobacter urease test (HUT) as a gold standard in a cross-sectional study of patients.

\section{Methods}

The study was conducted at the Endoscopy Unit of Korle $\mathrm{Bu}$ Teaching Hospital. It is a referral hospital with over 1800 beds for in-patients and has several specialist clinics, wards, pharmacies and reference laboratories. The study protocol was approved by Ethical and Protocol Review Committee of the School of Medicine and Dentistry, University of Ghana and all participating patients or their relatives provided written informed consent.

Seventy-six patients between 16 and 81 years referred for upper gastrointestinal endoscopy with dyspeptic symptoms were recruited for this study. Baseline bio-data were obtained from all patients and diagnosis of gastric disorders were made based on the presence of atrophic mucosa, oedematous mucosa, red spots or streaks and erosions suggesting inflammation and active or healing ulcers. Endoscopic diagnosis was made at the discretion of the endoscopist. Gastric antral mucosal biopsies of adequate size were then taken for $H$. pylori urease testing (HelicotecUT ${ }^{\circledR}$ Plus, Emergo Europe). In regions of atrophic gastritis, the biopsies were taken more proximally.

\section{Helicobacter urease test (HelicotecUT ${ }^{\circledR}$ Plus)}

Biopsy samples, approximately $2-3 \mathrm{~mm}$ each were taken from the antralgastric mucosa and placed on the yellow colored well containing urea and a $\mathrm{pH}$ indicator. The production of the urease enzyme by $H$. pylori results in the decomposition of urea into bicarbonate and ammonia which causes the $\mathrm{pH}$ to rise and the colour of the dot to change from yellow to red or pink. Positive results were read within 5 to $30 \mathrm{~min}$. Samples that were weakly positive took up to $1 \mathrm{~h}$ to develop and no colour change at $1 \mathrm{~h}$ was regarded negative.

\section{Statistical analysis}

All data were entered into Statistical Package for Social Science (ver.17.0; SPSS, Chicago, IL) and imported into Stata $^{\mathrm{TM}}$ version 10 (StataCorp, College Station, Texas, United States) for statistical analyses. Data were summarized as frequencies and proportions.

\section{Results}

A total of 76 patients were recruited for the study of which $43(56.6 \%)$ were females and $33(43.4 \%)$ were males. The median age of the study participants was 45.0 years [IQR 30.5-63.5]. A vast majority of the study subjects $(88.2 \%, \mathrm{n}=67)$ were from urban areas and 9 $(11.8 \%)$ were from rural areas. Severity of symptoms was assessed to be mild in $9(12.0 \%)$ and moderate to severe in $66(88 \%)$ of the patients. Endoscopic diagnosis was positive for H. pylori in 51 patients (67.1\%). However, the overall prevalence of $H$. pylori using the HUT among the recruited patients was $51.3 \%$ [ $95 \%$ CI 40.0-63.0].

Table 1 shows the distribution of the various symptoms reported by the patients. A total of 39 patients recruited in the study were positive for $H$. pylori urease test. The 
Table 1 Frequency of symptoms of patients attending endoscopy clinic at the Korle Bu Teaching Hospital in Accra, Ghana

\begin{tabular}{|c|c|c|c|c|}
\hline \multirow[t]{2}{*}{ Symptom } & \multicolumn{2}{|c|}{ H. pylori urease test results } & \multicolumn{2}{|c|}{ Endoscopic diagnosis results } \\
\hline & Positive $\mathrm{N}=39 \mathrm{n}, \%^{\mathrm{a}}$ & Negative $N=37 n, \%^{a}$ & Positive $\mathrm{N}=51 \mathrm{n}, \%^{\mathrm{a}}$ & Negative $N=25 n, \%^{a}$ \\
\hline Epigastric pain & $29(74.4)$ & $22(59.5)$ & $35(68.6)$ & $16(64.0)$ \\
\hline Nausea & $3(7.7)$ & $0(0)$ & $2(3.9)$ & $1(4.0)$ \\
\hline Vomiting & $1(2.6)$ & $0(0)$ & $1(2.0)$ & $0(0)$ \\
\hline Dyspepsia & $9(23.1)$ & $5(13.5)$ & $7(13.7)$ & $7(28.0)$ \\
\hline Chest pain & $7(17.9)$ & $6(16.2)$ & $9(17.6)$ & $4(16.0)$ \\
\hline Hematemesis & $4(10.3)$ & $8(21.6)$ & $8(15.7)$ & $4(16.0)$ \\
\hline Malena & $3(7.7)$ & $9(24.3)$ & $8(15.7)$ & $4(16.0)$ \\
\hline Regurgitation & $1(2.6)$ & $4(10.8)$ & $3(5.9)$ & $2(8.0)$ \\
\hline
\end{tabular}

a $\%$ s may not add up to 100 as one patient be diagnosed with more than one endoscopic finding

most reported symptom by patients was epigastric pain $(67.1 \%, \mathrm{n}=51)$. Among these patients reporting with epigastric pain, $74.4 \%(\mathrm{n}=29)$ tested positive for $H$. pylori urease test. For patients reporting with dyspepsia $23.1 \%(\mathrm{n}=9)$ were positive for HUT (Table 1$)$.

Table 2 shows the various endoscopy findings. The most frequent endoscopy finding was gastritis $(59.2 \%$, $\mathrm{n}=45)$. Seventeen patients $(22.4 \%)$ had normal endoscopy results. A total of 26 (66.7\%) of the patients who tested positive to HUT were diagnosed with gastritis whilst $8(20.5 \%)$ were diagnosed with gastric ulcer (Table 2).

Table 3 shows the association between the various exposure characteristics and HUT. In the univariate analysis, age grouping, dwelling status, endoscopy findings and endoscopy $H$. pylori diagnosis were not associated with HUT $(\mathrm{p}>0.05)$. In the multivariate analysis, gender and symptom severity were associated with $H$. pylori urease test results. The odds of a female showing positive test results for HUT was 2.73 times compared with males
(OR 2.73 [95 \% CI 1.02-7.33], p = 0.0460) although this association is marginal (Table 3). Symptom severity was associated with HUT results such that the odds of a patient reporting with moderate to severe symptoms having a positive HUT test results was 12.06 times (OR 12.06 [95 \% CI 1.38-105.59], $\mathrm{p}=0.024$ ) compared with patients who reported with mild symptoms (Table 3 ).

Table 4 shows the various medications that the patients were taking and how it related to the $H$. pylori status as detected by HUT and by endoscopic diagnosis. $18 \%$ of patients who admitted to taking PPI's were HUT positive while $51 \%$ were negative.

Diagnostic yield of endoscopic diagnosis is $55.3 \%$. Using endoscopic diagnosis as the index test and HUT as the reference standard, the sensitivity and specificity of the index test were $71.8 \%$ [95 \% CI 55.1-85.0] and $37.8 \%$ [95 \% CI 22.5-55.2] respectively. Further analysis indicated that the area under the receiver operated characteristics curve was 0.55 [95\% CI 0.44-0.65] indicating that the endoscopic diagnosis test do not predict $H$.

Table 2 Frequency of endoscopy findings of patients attending endoscopy clinic at the Korle Bu Teaching Hospital in Accra, Ghana

\begin{tabular}{|c|c|c|c|c|}
\hline \multirow[t]{2}{*}{ Endoscopic finding } & \multicolumn{2}{|c|}{ H. pylori urease test results } & \multicolumn{2}{|c|}{ Endoscopic diagnosis results } \\
\hline & $\begin{array}{l}\text { Positive } N=39 \\
\text { n, \% }\end{array}$ & $\begin{array}{l}\text { Negative } N=37 \\
n, \%^{a}\end{array}$ & $\begin{array}{l}\text { Positive } N=51 \\
\text { n, } \%^{a}\end{array}$ & $\begin{array}{l}\text { Negative } N=25 \\
n, \%^{a}\end{array}$ \\
\hline Gastritis & $26(66.7)$ & $19(51.4)$ & $40(78.4)$ & $5(20.0)$ \\
\hline Gastric ulcer & $2(5.1)$ & $4(10.8)$ & $6(11.7)$ & $0(0)$ \\
\hline Duodenal ulcer & $6(15.4)$ & $4(10.8)$ & $10(19.6)$ & $0(0)$ \\
\hline Gastric polyp & $0(0)$ & $1(2.7)$ & $0(0)$ & $1(4.0)$ \\
\hline Normal & $6(15.4)$ & $11(29.7)$ & $2(3.9)$ & $15(60.0)$ \\
\hline Candidiasis & $0(0)$ & $1(2.7)$ & $1(2.0)$ & $0(0)$ \\
\hline Reflux esophagitis & $1(2.6)$ & $0(0)$ & $1(2.0)$ & $0(0)$ \\
\hline Oesophageal stricture & $0(0)$ & $1(2.7)$ & $1(2.0)$ & $0(0)$ \\
\hline
\end{tabular}

\footnotetext{
a $\%$ s may not add up to 100 as one patient be diagnosed with more than one endoscopic finding
} 
Table 3 Factors associated with positive $\boldsymbol{H}$. pylori urease test

\begin{tabular}{|c|c|c|c|c|c|c|}
\hline \multirow[t]{2}{*}{ Characteristic } & \multicolumn{2}{|c|}{ H. pylori urease test } & \multirow[t]{2}{*}{ Crude OR [95 \% Cl] } & \multirow[t]{2}{*}{$\mathrm{p}$ value } & \multirow{2}{*}{$\begin{array}{l}\text { Adjusted OR } \\
{[95 \% \mathrm{Cl}]}\end{array}$} & \multirow[t]{2}{*}{ p value } \\
\hline & Positive n, \% & Negative $\mathrm{n}, \%$ & & & & \\
\hline \multicolumn{7}{|l|}{ Gender } \\
\hline Female & $26(66.7)$ & $17(46.0)$ & 2.35 [0.93-5.95] & 0.069 & $2.73[1.02-7.33]$ & 0.046 \\
\hline Male & $13(33.3)$ & $20(54.1)$ & Ref & & Ref & \\
\hline \multicolumn{7}{|l|}{ Age group } \\
\hline$<20$ & $1(2.6)$ & $1(2.7)$ & $1.33[0.07-24.32]$ & 0.846 & - & - \\
\hline $20-29$ & $9(23.1)$ & $8(21.6)$ & $1.50[0.41-5.43]$ & 0.537 & - & - \\
\hline $30-39$ & $9(23.1)$ & $5(13.5)$ & $2.40[0.60-9.67]$ & 0.218 & - & - \\
\hline $40-49$ & $4(10.3)$ & $7(18.9)$ & $0.76[0.17-3.42]$ & 0.723 & - & - \\
\hline $50-59$ & $7(18.0)$ & $4(10.8)$ & $2.33[0.52-10.48]$ & 0.269 & - & - \\
\hline$\geq 60$ & $9(23.1)$ & $12(32.4)$ & Ref & & & \\
\hline \multicolumn{7}{|l|}{ Dwelling } \\
\hline Rural & $4(10.3)$ & $5(13.9)$ & $0.71[0.13-3.63]$ & 0.629 & - & - \\
\hline Urban & 35 (89.7) & $31(86.1)$ & Ref & & & \\
\hline \multicolumn{7}{|l|}{ Symptom severity } \\
\hline Moderate/severe & $38(96.4)$ & $28(77.8)$ & 10.86 [1.29-94.05] & 0.009 & 12.06 [1.38-105.59] & 0.024 \\
\hline Mild & $1(2.6)$ & $8(22.2)$ & Ref & & Ref & \\
\hline \multicolumn{7}{|l|}{ Endoscopy finding } \\
\hline Abnormal & $28(73.7)$ & $28(77.8)$ & $0.80[0.24-2.64]$ & 0.682 & - & - \\
\hline Normal & $10(26.3)$ & $8(22.2)$ & Ref & & & \\
\hline \multicolumn{7}{|c|}{ Endoscopic diagnosis } \\
\hline Positive & $28(71.8)$ & $23(62.2)$ & 1.55 [0.59-4.06] & 0.373 & - & - \\
\hline Negative & $11(28.2)$ & $14(37.8)$ & Ref & & & \\
\hline
\end{tabular}

Table 4 Comparison of medications with $H$. pylori urease test and with the endoscopic diagnosis

\begin{tabular}{|c|c|c|c|c|}
\hline \multirow[t]{2}{*}{ Medication } & \multicolumn{2}{|c|}{ HUT results } & \multicolumn{2}{|c|}{ Endoscopic findings } \\
\hline & $\begin{array}{l}\text { Positive } \\
\text { (\%) }\end{array}$ & $\begin{array}{l}\text { Negative } \\
\text { (\%) }\end{array}$ & $\begin{array}{l}\text { Positive } \\
\text { (\%) }\end{array}$ & $\begin{array}{l}\text { Negative } \\
(\%)\end{array}$ \\
\hline \multicolumn{5}{|l|}{ PPI } \\
\hline Yes & $7(18.0)$ & $19(51.4)$ & $14(27.5)$ & $12(48.0)$ \\
\hline No & $32(82.0)$ & $18(48.6)$ & $37(72.5)$ & $13(52.0)$ \\
\hline \multicolumn{5}{|l|}{ Antacid } \\
\hline Yes & $9(23.1)$ & $8(21.6)$ & $14(27.5)$ & $3(12.0)$ \\
\hline No & $30(76.9)$ & $29(78.4)$ & $37(72.5)$ & $22(88.0)$ \\
\hline \multicolumn{5}{|l|}{$\mathrm{H}_{2}$ blocker } \\
\hline Yes & $1(2.6)$ & $0(0)$ & $1(2.0)$ & $0(0)$ \\
\hline No & $38(97.4)$ & $37(100)$ & $50(98.0)$ & $25(100)$ \\
\hline \multicolumn{5}{|l|}{ NSAID } \\
\hline Yes & $13(33.3)$ & $8(21.6)$ & $17(33.3)$ & $4(16.0)$ \\
\hline No & $26(66.7)$ & $29(78.4)$ & $34(66.7)$ & $21(84.0)$ \\
\hline
\end{tabular}

pylori status (as determined by HUT) better than chance. After excluding those on PPI, diagnostic yield was $58 \%$, sensitivity of 75.03 [95 \% CI 56.6-88.5] and specificity of $38.8 \%$ [95 \% CI 13.9-68.4]. The area under the receiver operated characteristics curve was 0.51 [95\% CI
0.37-0.66] implying that in patients who have not been exposed to PPI endoscopic diagnosis test do not predict H. pylori status.

\section{Discussion}

The most common symptoms leading to referral for upper GI endoscopy were epigastric pain, dyspepsia, upper gastrointestinal bleeding (haematemesis and melaena) and chest pain respectively which is the same as that reported in earlier publications [3]. The current study found the incidence of $H$ pylori infection in Korle $\mathrm{Bu}$ Teaching Hospital to be $51.3 \%$ based on the HUT. The prevalence is much lower than that quoted in other studies from West Africa [3, 4, 9] which have shown a high prevalence of $H$. pylori. Previous studies in Korle $\mathrm{Bu}$ Teaching Hospital reported an incidence of $75 \%[3,4]$. However, a recent publication also reports an $H$. pylori prevalence of $69.7 \%$ (1999) and $45.2 \%$ (2012) in Accra [12]. This suggests a change in $H$. pylori prevalence with time.

Gastritis (59.2 \%) was the most common identifiable lesion at endoscopy in this study. One study done in Tanzania with $H$. pylori prevalence of $65 \%$ also reported gastritis as the most common finding and gastritis and duodenal ulcer were statistically associated with $H$. 
pylori. $72 \%$ of gastritis and $89.5 \%$ of duodenal ulcers were $H$. pylori positive [13]. Based on previous prevalence studies, it has been established that the finding of a non-bleeding duodenal ulcer has a positive predictive value of over $90 \%$ for $H$. pylori diagnosis, making confirmatory tests not mandatory. However, the positive predictive values for gastric ulcers, bleeding duodenal ulcers and perforated duodenal ulcers are lower and confirmatory tests would be necessary [14].

Nearly $58 \%$ of gastritis and $60 \%$ of duodenal ulcers tested positive for $\mathrm{H}$. pylori, while $33 \%$ of gastric ulcers were also positive. Interestingly our study did not find any positive association between duodenal ulcers, gastric ulcers or gastritis and a positive HUT. This may be explained by the fact that, among the 10 patients with duodenal ulcer, 3 of the 4 patients who were HUT negative admitted to using PPI's. While of the 6 patients diagnosed with gastric ulcer, 3 of the 4 who had negative HUT admitted to using PPI's. Cohen et al. [14] have reported that the use of PPI's may lead to unreliable $H$ pylori diagnosis.

Moderate to severe symptoms were associated with HUT results such that the odds of a patient reporting with moderate to severe symptoms of having a positive HUT test result was 12.06 times (OR 12.06 [95 \% CI 1.38-105.59], $\mathrm{p}=0.024$ ) compared with patients who reported with mild symptoms. This means endoscopy with $H$. pylori diagnosis and treatment is mandatory in these patients whereas those with mild symptoms may be managed without $H$. pylori testing.

The gold standard recommended by the World Gastroenterology Organisation Global Guidelines, 2010 is endoscopy and rapid urease test which may not be readily available or cost effective in developing countries. They recommend then that in resource limited settings with a high prevalence, the decision to treat may be based on the assumption that $H$. pylori is present. This has been the practice over the years, however, recent development has seen the introduction of several endoscopy set ups in both public and private facilities. This has brought with it increased opportunities for the accurate diagnosis and treatment of $\mathrm{H}$. pylori. Accurate diagnosis and treatment is necessary if the prevalence is not as high as previously reported. The empirical treatment with antibiotics based on endoscopic diagnosis and assumption of high prevalence should be discouraged. Efforts should be made to make an accurate diagnosis of $H$. pylori before treatment. Where the HUT is not available other tests should be done such as histological diagnosis or stool for H. pylori antigen. The lower prevalence we report may be due to improving standards of living and sanitation in Ghana as compared to previous studies published several years ago also using HUT [3, 4]. In addition the vast majority of our study subjects were from urban areas where the standard of living is higher. However, the frequent use of antibiotics may also contribute to the relatively low prevalence being reported recently [12]. Also the recent use of PPI's may contribute to the low prevalence. Of all the patients who admitted to taking PPI's only $18 \%$ had positive HUT as compared to $51 \%$ who tested negative. While for those who denied PPI use, $82 \%$ had positive HUT and $49 \%$ were negative. This supports the fact that recent use of PPI's may result in falsely negative HUT results [14].

With regards to the endoscopic diagnosis this study reports a diagnostic yield of $55.3 \%$ with a sensitivity and specificity of $71.8 \%$ [95 \% CI 55.1-85.0] and 37.8 \% [95\% CI 22.5-55.2] respectively, indicating that the endoscopic diagnosis does not predict $H$. pylori status (as determined by HUT) better than chance. After excluding those on PPI, analysis resulted in a diagnostic yield of $58 \%$, sensitivity of 75.03 [95 \% CI 56.6-88.5] and specificity of $38.8 \%$ [95 \% CI 13.9-68.4]. The value of endoscopic diagnosis of $H$. pylori is unclear. The value of the HUT in the patients who have recently been on PPIs and antibiotics maybe limited. In this case treatment based on the endoscopic diagnosis should be considered as recommended by WHO. On the other hand in treatment naive patients, the HUT maybe more reliable and treatment can be prescribed when indicated.

It is known that certain endoscopic mucosal features indicate $H$. pylori infection such as atrophic changes, rugal hyperplasia, oedema, spotty erythema, linear erythema and haemorrhage, amongst others. However, recognition and diagnosis depends on the experience of the endoscopist. Several studies including that by Khaloo et al. (41.8\%) [15] and Redeen et al. (43-53\%) [16] have reported a low diagnostic yield. Khazuhiro et al. however, published a relatively higher diagnostic yield in the $H$. pylori-uninfected ( $88.9 \%$ ) but lower in $H$. pylori-infected (62.1\%) and in the $H$. pylori-eradicated $(55.8 \%)$ patients [17]. This current study, like others, does not support endoscopic diagnosis of $H$. pylori as a standard of care.

Magnifying endoscopy affords the opportunity to make an accurate endoscopic diagnosis of $H$. pylori based on the surface structure of the gastric mucosa and has sensitivity and specificity of 100 and $92.7 \%$ respectively for $H$. pylori infected mucosa [18]. This facility is not available at many endoscopy centers worldwide and not in Ghana and other developing countries. It may now be cost effective to make an accurate diagnosis before treatment so as to cut down cost on antibiotics, its side effects and reduce antibiotic resistance.

The obvious advantage of the rapid urease test in our environment is that it is inexpensive (less than $\$ 10$ / test), does not require any technical expertise and gives rapid results within an hour. It is also reliable with the 
sensitivity of Campylobacter-like organism (CLO) test $75-98 \%$ and specificity 95-100 \% [14]. This will improve the accurate diagnosis especially where endoscopic expertise is not certain.

With histologic diagnosis the disadvantages are sampling errors, observer variations, high cost and long duration of processing. There is also an additional delay in our facility as the patient is responsible for getting the specimen to the pathologist and getting the report back to the physician.

\section{Limitation}

One of the limitations of our study is taking antral biopsy samples. We also did not have the sufficient resources to recruit more patients, which may have improved the statistical power of our studies. This study was also undertaken in a single medical center, Korle Bu Teaching Hospital, which may not be a general representation of Ghana although the patients came from variety of ethnic groups and socioeconomic backgrounds.

\section{Conclusion}

The incidence of $H$. pylori infection in Ghana may be lower than previously reported due to changing socioeconomic factors, increased PPI and antibiotic use. To determine the true incidence of $H$. pylori infection in Ghana a larger study cutting across all the socio-economic classes needs to be done on a treatment naive population. The endoscopic diagnosis alone of $H$. pylori infection is not sufficient as it has a low sensitivity and specificity and depends on the experience of the endoscopist. The rapid HUT is simple and inexpensive and should be introduced routinely at all endoscopy centers in Ghana and other developing countries. Endoscopists at these centers should be educated on its interpretation based on patient's drug history.

\section{Abbreviations}

H. pylori: Helicobacter pylori; HUT: Helicobacter urease test; Cl: confidence interval; CLO: Campylobacter-like organism; OR: odd ratio; DNA: deoxyribonucleic acid; PCR: polymerase chain reaction; UBT: urea breath tests; KBTH: Korle Bu Teaching Hospital.

\section{Authors' contributions}

The study was conceived and designed by NAA and LA.JN, FD and NAA performed the endoscopy and WK assisted with the HUT. WK and LA assisted in interpreting the results and drafting the manuscript. All authors read and approved the final manuscript.

\section{Author details \\ ${ }^{1}$ Department of Surgery, School of Medicine and Dentistry, College of Health Sciences, P.O. Box GP 4236, Accra, Ghana. ${ }^{2}$ Division of Gastroenterology, University of Oslo, Oslo, Norway. ${ }^{3}$ Centre for Tropical Clinical Pharmacology and Therapeutics, School of Medicine and Dentistry, College of Health Sci- ences, P.O. Box GP 4236, Accra, Ghana.}

\section{Acknowledgements}

The authors would like to express their appreciation to Mr Edmund Nartey of School of Medicine and Dentistry, College of Health Sciences, for statistical analysis of the data.

\section{Competing interests}

The authors declare that they have no competing interests.

\section{Ethical approval}

The study protocol was approved by Ethical and Protocol Review Committee of the School of Medicine and Dentistry, University of Ghana and all participating patients or their relatives provided written informed consent.

Received: 4 December 2015 Accepted: 23 August 2016

Published online: 30 August 2016

References

1. Malfertheiner P, Megraud F, O'Morain C, Bazzoli F, El-Omar E, Graham D, Hunt R, Rokkas T, Vakil N, Kuipers EJ. Current concepts in the management of Helicobacter pylori infection: the Maastricht III Consensus Report. Gut. 2007;56(6):772-81.

2. Kidd M, Lastovica AJ, Atherton JC, Louw JA. Heterogeneity in the Helicobacter pylori vacA and cagA genes: association with gastroduodenal disease in South Africa? Gut. 1999;45(4):499-502.

3. Aduful H, Naaeder S, Darko R, Baako B, Clegg-Lamptey J, Nkrumah K, Adu-Aryee N, Kyere M. Upper gastrointestinal endoscopy at the Korle Bu Teaching Hospital, Accra, Ghana. Ghana Med J. 2007;41(1):12-6.

4. Baako BN, Darko R. Incidence of Helicobacter pylori infection in Ghanaian patients with dyspeptic symptoms referred for upper gastrointestinal endoscopy. West Afr J Med. 1996;15(4):223-7.

5. Graham KS, Ingram JD, Steinberg SE, Narkewicz MR. ERCP in the management of pediatric pancreatitis. Gastrointest Endosc. 1998;47(6):492-5.

6. Kusters JG, van Vliet AH, Kuipers EJ. Pathogenesis of Helicobacter pylori infection. Clin Microbiol Rev. 2006:19(3):449-90.

7. Segal I, Ally R, Mitchell H. Gastric cancer in sub-Saharan Africa. Eur J Cancer Prev. 2001:10(6):479-82.

8. Diomande MI, Flejou JF, Potet F, Dago-Akribi A, Ouattara D, Kadjo K, Niamkey E, Beaumel A, Gbe K, Beda BY. Chronic gastritis and Helicobacter pylori infection on the Ivory Coast. A series of 277 symptomatic patients. Gastroenterol Clin Biol. 1991:15(10):711-6.

9. Jemilohun AC, Otegbayo JA, Ola SO, Oluwasola OA, Akere A. Prevalence of Helicobacter pylori among Nigerian patients with dyspepsia in Ibadan. Pan Afri Med J. 2010;6:18.

10. Jones N, Chiba N, Fallone C, Thompson A, Hunt R, Jacobson K, Goodman K. Helicobacter pylori in first nations and recent immigrant populations in Canada. Can J Gastroenterol. 2012;26(2):97-103.

11. Roma-Giannikou E, Roubani A, Sgouras DN, Panayiotou J, van Vliet C, Polyzos A, Roka K, Daikos G. Endoscopic tests for the diagnosis of Helicobacter pylori infection in children: validation of rapid urease test. Helicobacter. 2010;15(3):227-32.

12. Darko RYA, Osei V, Owusu-Ansah J, Aluze-Ele S. Changing partterns of the prevalence of Heleicobacter pylori among patients at a coorporate hospital in Ghana. Ghana Med J. 2015;49(3):147-53.

13. Ayana SM, Swai B, Maro VP. Upper gastrointestinal endoscopic findings and prevalence of Helicobacter pylori infection among adult patients with dyspepsia in northern Tanzania. Tanzan J Health Res. 2014;16(1):16-22.

14. Cohen H, Laine L. Endoscopic methods for the diagnosis of Helicobacter pylori. Aliment Pharmacol Ther. 1997;11(Suppl 1):3-9.

15. Khakoo SI, Lobo AJ, Shepherd NA, Wilkinson SP. Histological assessment of the sydney classification of endoscopic gastritis. Gut. 1994;35(9):1172-5.

16. Redeen S, Petersson F, Jonsson KA, Borch K. Relationship of gastroscopic features to histological findings in gastritis and Helicobacter pylori infection in a general population sample. Endoscopy. 2003;35(11):946-50.

17. Watanabe K, Nagata N, Shimbo T, Nakashima R, Furuhata E, Sakurai T, Akazawa N, Yokoi C, Kobayakawa M, Akiyama J, et al. Accuracy of endoscopic diagnosis of Helicobacter pylori infection according to level of endoscopic experience and the effect of training. BMC Gastroenterol. 2013;13:128.

18. Kim S, Ito M, Haruma K, Egi Y, Ueda H, Tanaka S, Chayama K. Surface structure of antral gastric mucosa represents the status of histologic gastritis: fundamental evidence for the evaluation of antral gastritis by magnifying endoscopy. J Gastroenterol Hepatol. 2006;21(5):837-41. 